Supporting Information for

\title{
Synthesis and Catalytic Properties of Porous Metal Silica Materials Templated and Functionalized by Extended Coordination Cages
}

\author{
Jiahui Ye, ${ }^{\mathrm{a}}$ Jiajun Tang, ${ }^{\mathrm{ab}}$ Yu-Jun Zhao, ${ }^{\mathrm{b}}$ and Chuan-De $\mathrm{Wu}^{\mathrm{ac} *}$ \\ ${ }^{a}$ School of Chemical Engineering and Light Industry, Guangdong University of Technology, Guangzhou \\ 510006, P. R. China \\ ${ }^{b}$ Department of Physics, South China University of Technology, Guangzhou 510640, P. R. China \\ 'State Key Laboratory of Silicon Materials, Department of Chemistry, Zhejiang University, Hangzhou \\ 310027, P. R. China
}




\section{2-Methylimidazole}

dissolved in $\mathrm{H}_{2} \mathrm{O}$

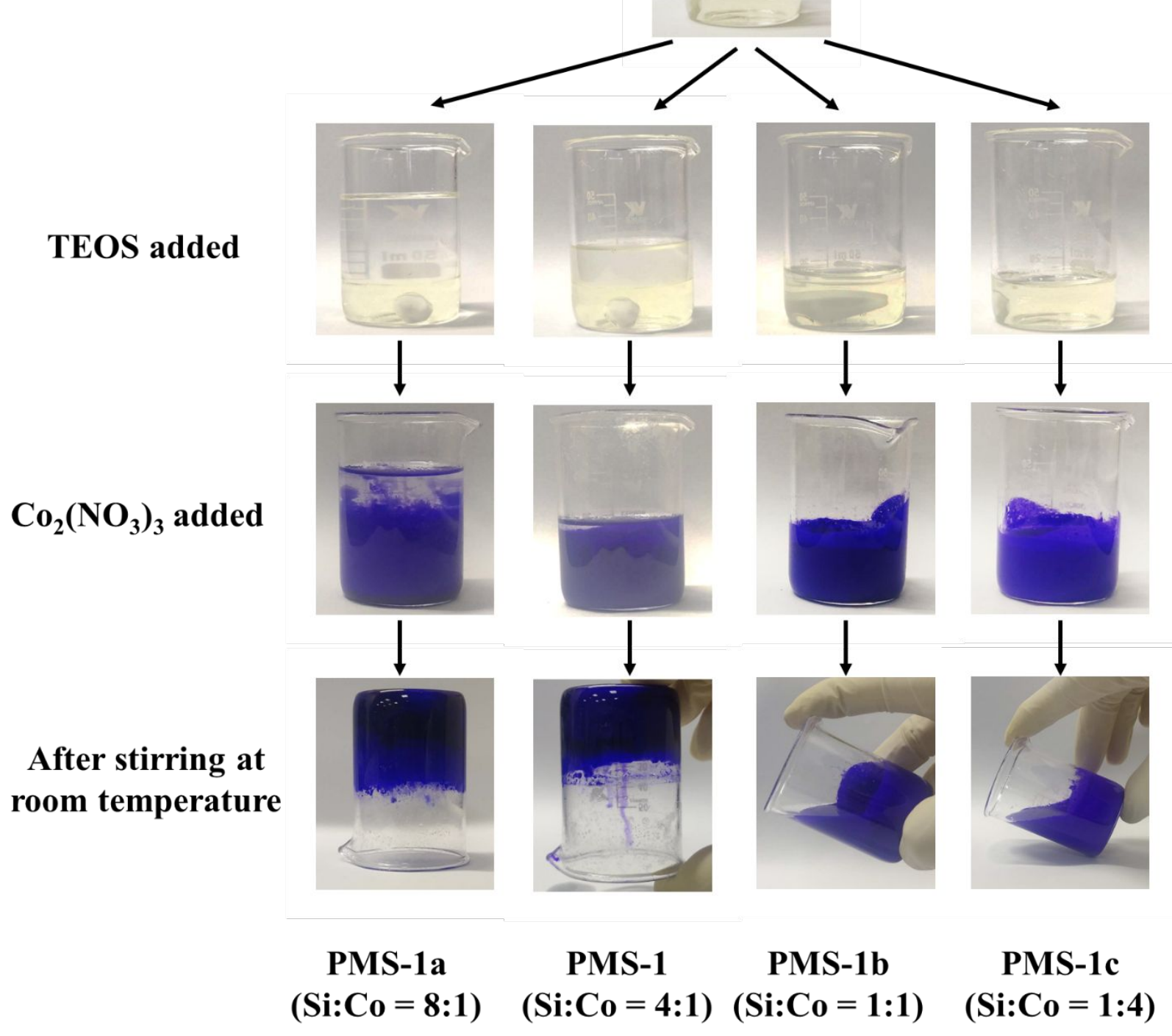

Figure S1. Schematic representation of the synthetic procedures for different PMS precursors, showing the color changes and congealing states of PMS precursors during preparation. 

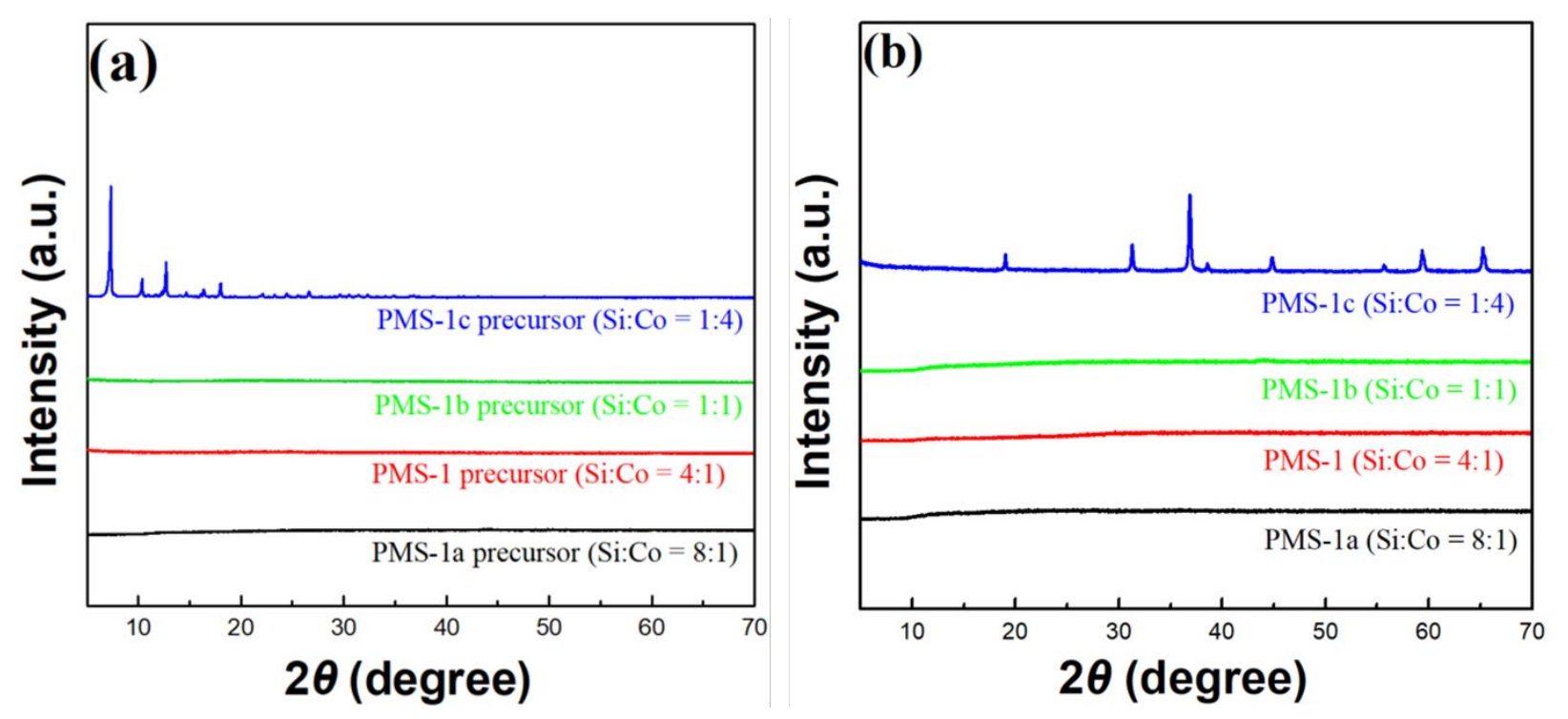

Figure S2. PXRD profiles of (a) before and (b) after calcination of the PMS precursor materials with different $\mathrm{Si} / \mathrm{Co}$ ratios.

The PMS materials were synthesized with various Si/Co ratios of 8:1 (PMS-1a), 4:1 (PMS-1), 1:1 (PMS-1b) and 1:4 (PMS-1c), according to the described synthesis method. Figure S2 indicates that the PMS materials with the Si/Co ratios of $1: 1,4: 1$ and $8: 1$ in the recipes are amorphous, and the calcined products are amorphous as well. The surface properties of calcined materials are listed in Table S3. The BET surface area climbs when the $\mathrm{Si} / \mathrm{Co}$ ratio increases from 1:4 $\left(45 \mathrm{~m}^{2} \mathrm{~g}^{-1}\right)$ to $4: 1\left(391 \mathrm{~m}^{2} \mathrm{~g}^{-1}\right)$, whereas the BET surface area slightly increases when the ratio was increased from 4:1 $\left(391 \mathrm{~m}^{2} \mathrm{~g}^{-1}\right)$ to $8: 1\left(409 \mathrm{~m}^{2} \mathrm{~g}^{-1}\right)$. 


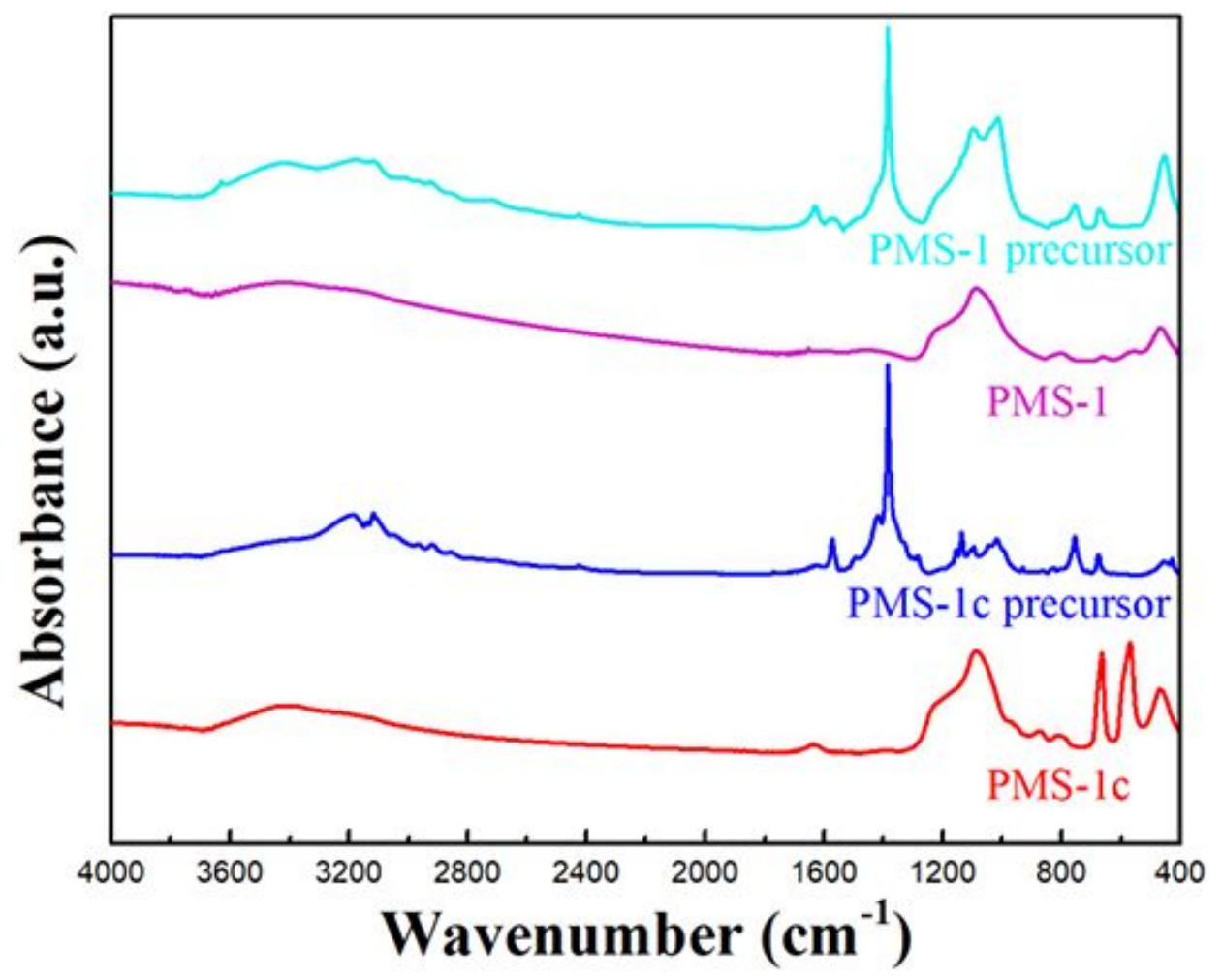

Figure S3. FT-IR spectra of PMS-1 precursor, PMS-1, PMS-1c precursor and PMS-1c. 


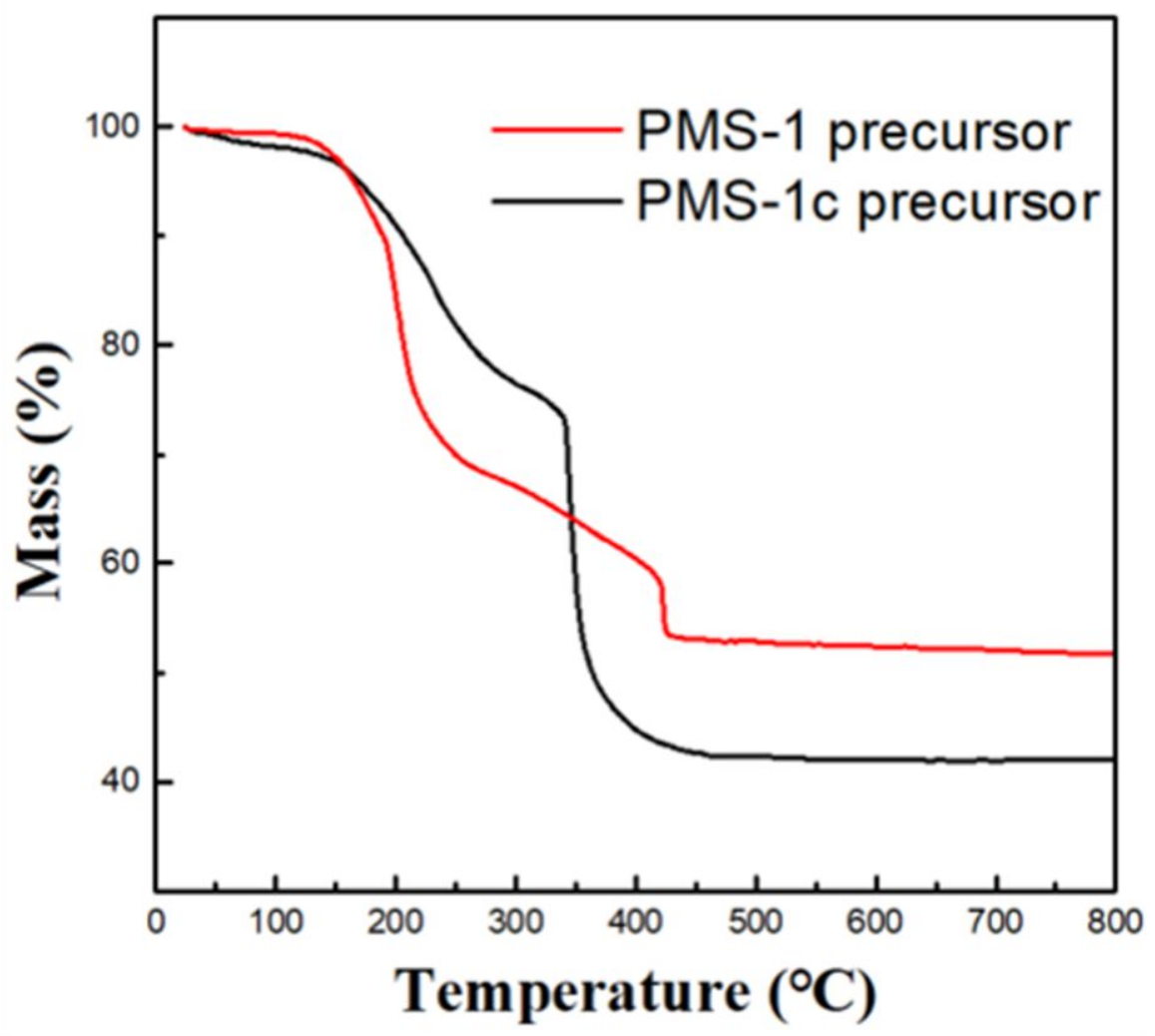

Figure S4. Comparison of the TG curves for PMS-1 precursor and PMS-1c precursor.

Figure S3 shows the FT-IR spectra of PMS-1/1c precursors and PMS-1/1c. The results indicate that the peak at $\sim 1600 \mathrm{~cm}^{-1}$ disappeared after calcination at $600{ }^{\circ} \mathrm{C}$, and the characteristic peaks for $\mathrm{Co}_{3} \mathrm{O}_{4}$ formed at 570 and $460 \mathrm{~cm}^{-1}$. Figure S4 shows the TG profiles of PMS-1 and PMS-1c precursors, indicating that the calcination temperature at $600{ }^{\circ} \mathrm{C}$ could entirely decompose the organic moieties in the precursors. 

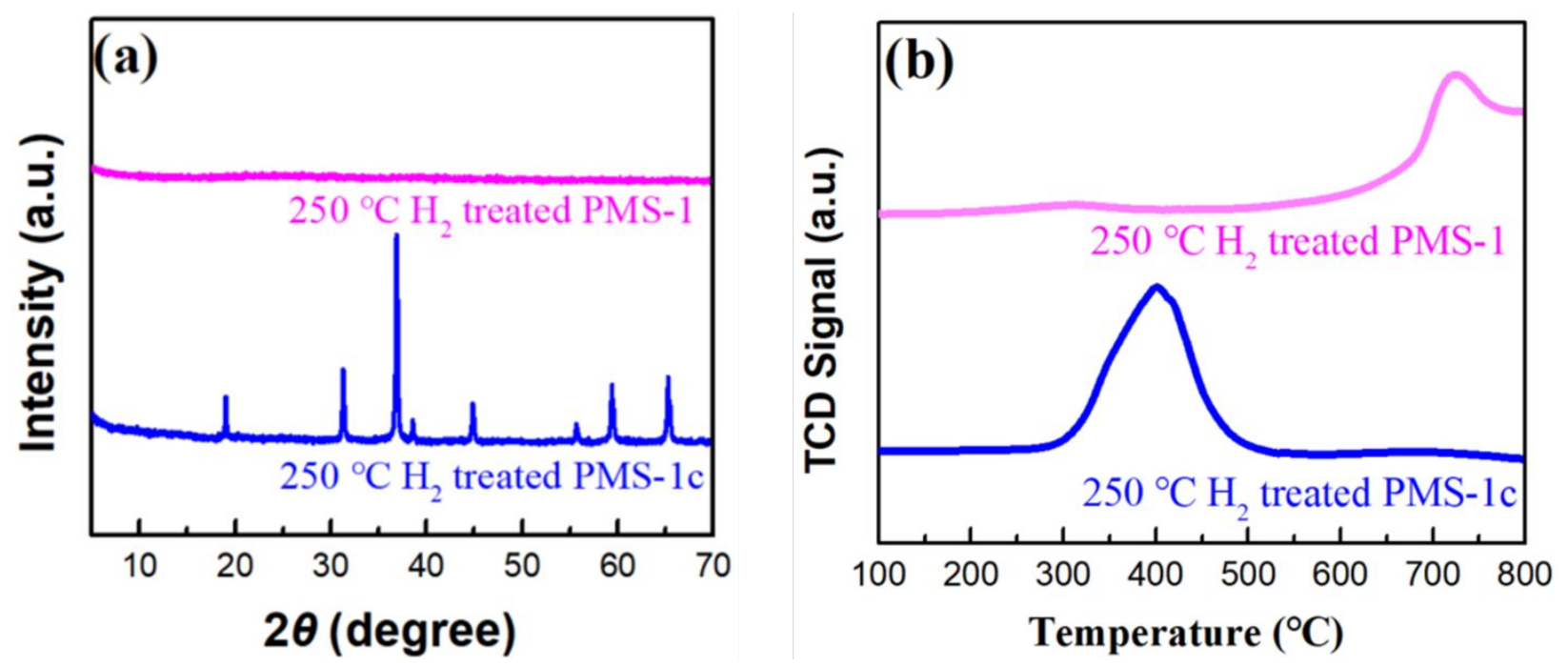

Figure S5. (a) PXRD and (b) $\mathrm{H}_{2}$-TPR profiles for $250{ }^{\circ} \mathrm{C} \mathrm{H}_{2}$ treated PMS-1 and $250{ }^{\circ} \mathrm{C} \mathrm{H}_{2}$ treated PMS-1c. The catalysts were treated with $10 \% \mathrm{H}_{2}$ in nitrogen at $250{ }^{\circ} \mathrm{C}$ for $2 \mathrm{~h}$. 

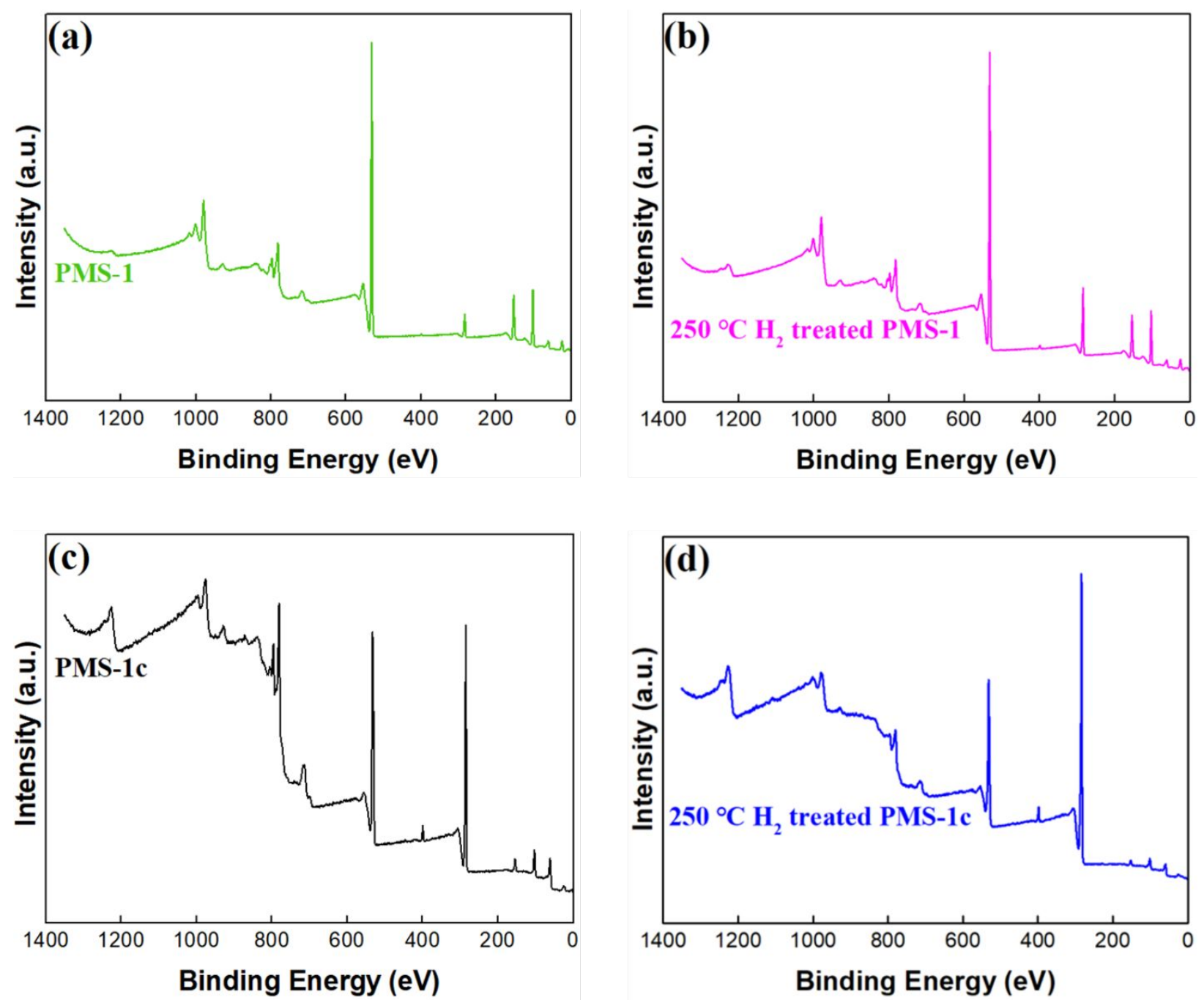

Figure S6. The XPS spectra of (a) PMS-1, (b) $250{ }^{\circ} \mathrm{C} \mathrm{H}_{2}$ treated PMS-1, (c) PMS-1c, and (d) $250{ }^{\circ} \mathrm{C} \mathrm{H}_{2}$ treated PMS- $1 \mathrm{c}$. 

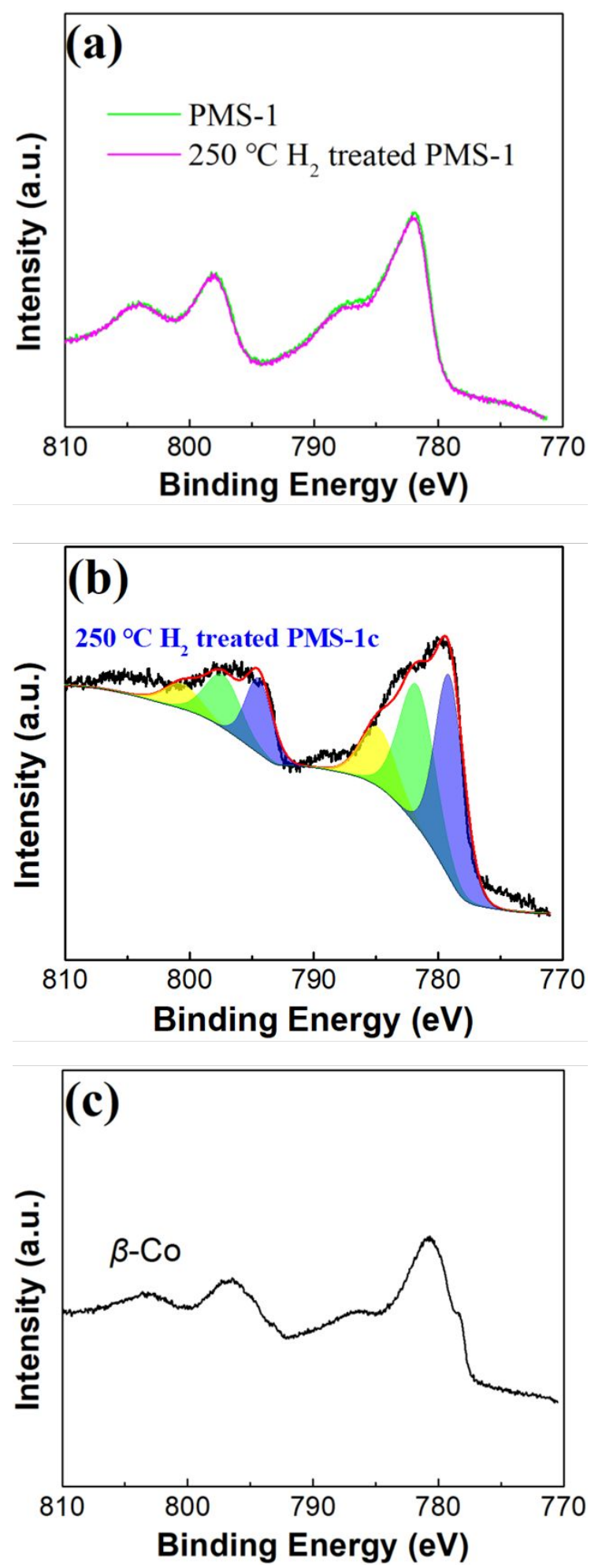

Figure S7. The Co $2 p$ XPS spectra of (a) $250{ }^{\circ} \mathrm{C} \mathrm{H}_{2}$ treated PMS-1, (b) $250{ }^{\circ} \mathrm{C} \mathrm{H}_{2}$ treated PMS$1 \mathrm{c}$, and (c) $\beta$-Co. 


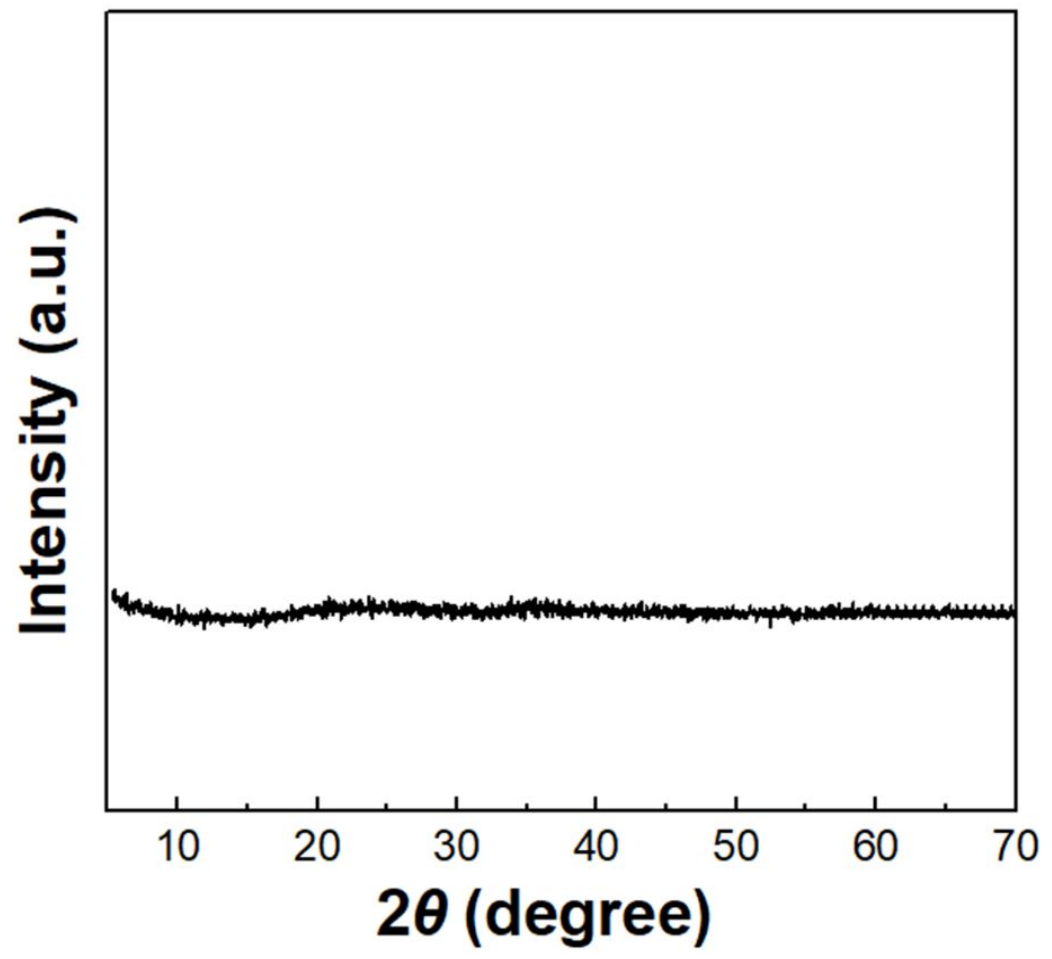

Figure S8. PXRD profile of PMS-1 after catalysis. 


\section{$\mathrm{Co}-\mathrm{O}=\mathrm{O}$ terminated surface}

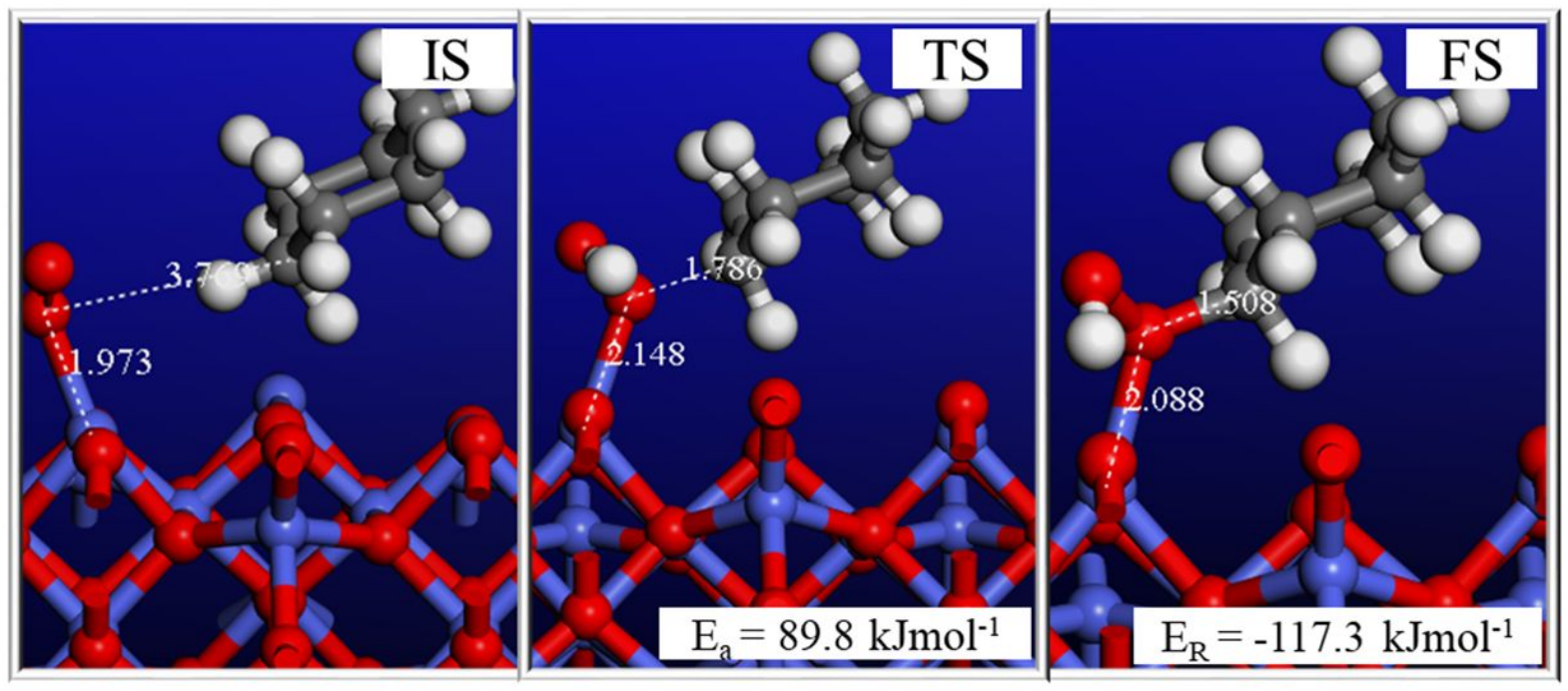

\section{$\mathrm{Co}-(-\mathrm{O}=\mathrm{O}-)$ terminated surface}
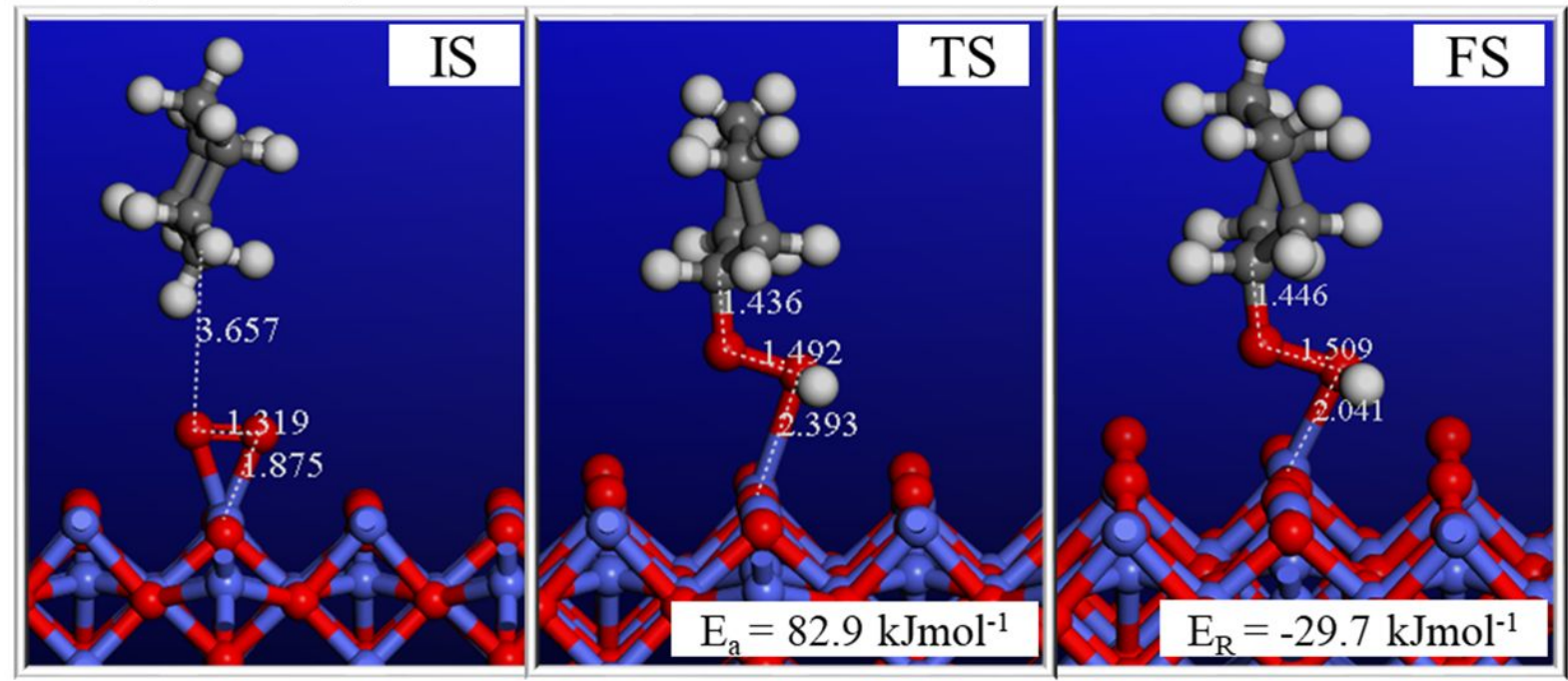

Figure S9. Activation barriers for the first C-H bond activation of cyclohexane over $\mathrm{Co}_{3} \mathrm{O}_{4}(110)$ surface terminated with $\mathrm{Co}-\mathrm{O}=\mathrm{O}$ and $\mathrm{Co}(-\mathrm{O}=\mathrm{O}-)$. The bond lengths are shown in white texts $(\AA)$. 


\section{$\mathrm{Co}^{-}(\mathrm{CyCOOH}) \longrightarrow \mathrm{Co}^{*}+\mathrm{CyCOOH}$}

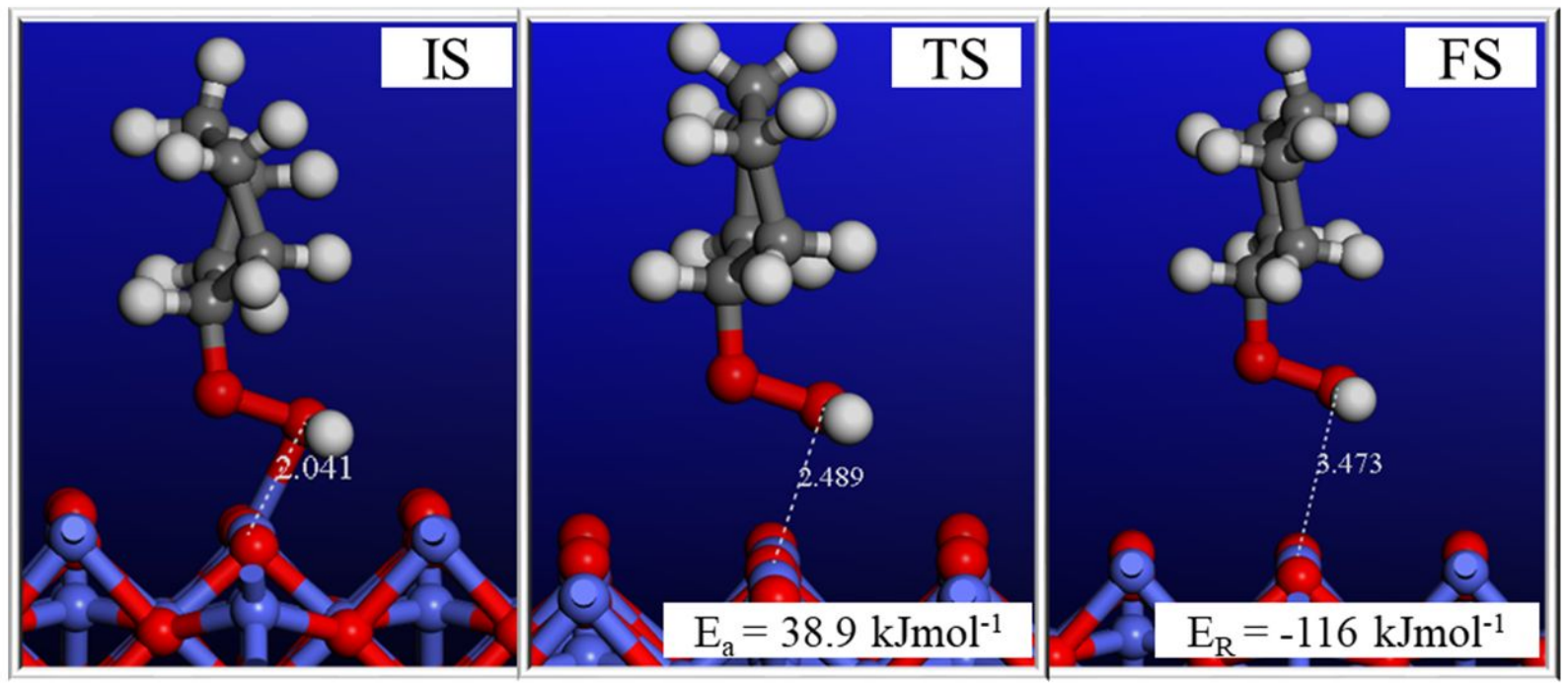

$\mathrm{Co}-(\mathrm{CyCOOH}) \longrightarrow \mathrm{Co}^{-} \mathrm{OH}+\mathrm{CyCO}$

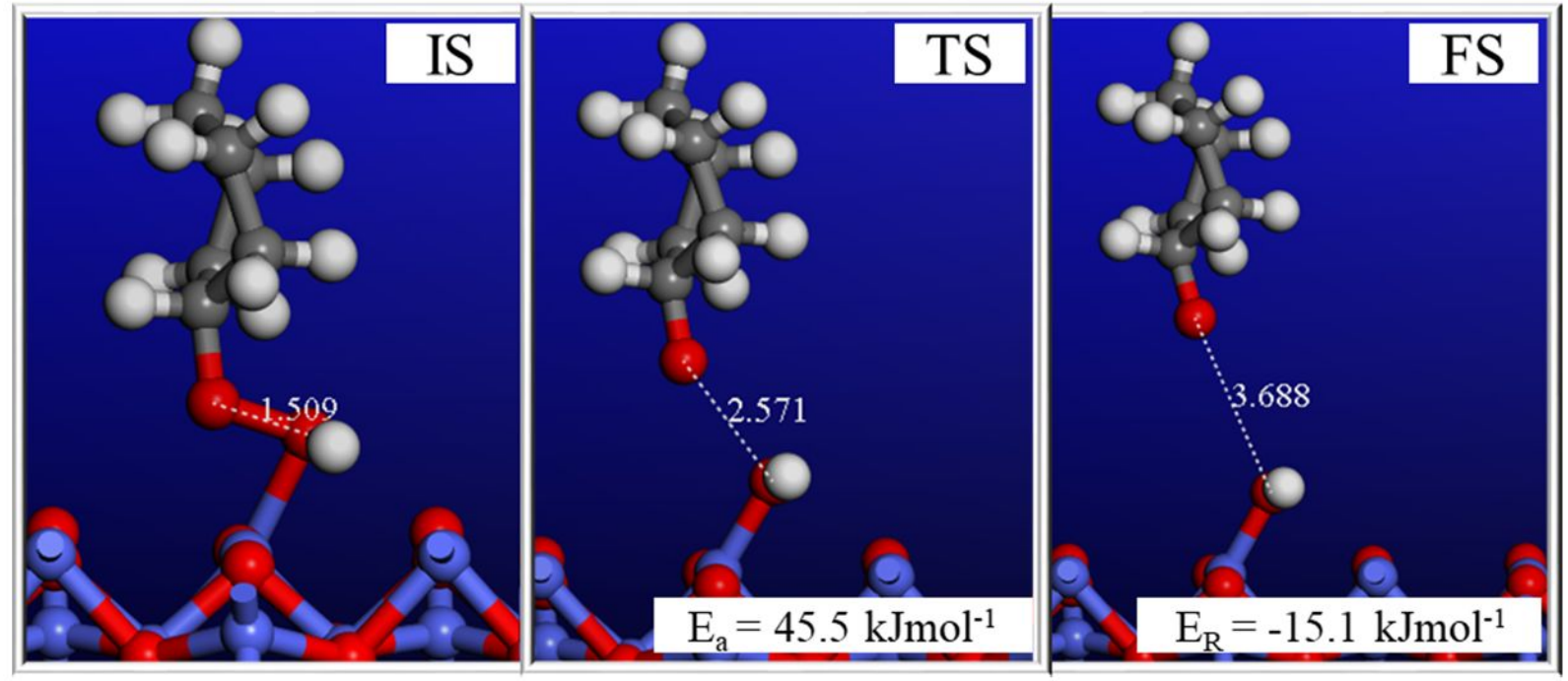

Figure S10. Activation barriers for the breakage of $\mathrm{Co}-(\mathrm{CyOOH})$ and the formation of $\mathrm{CyCOOH}$ or $\mathrm{CyCO} \cdot$ radical. The bond lengths are shown in white texts $(\AA)$. 


\section{Homolytic mechanism on flat $\mathrm{Co}_{3} \mathrm{O}_{4}(110)$ surface}

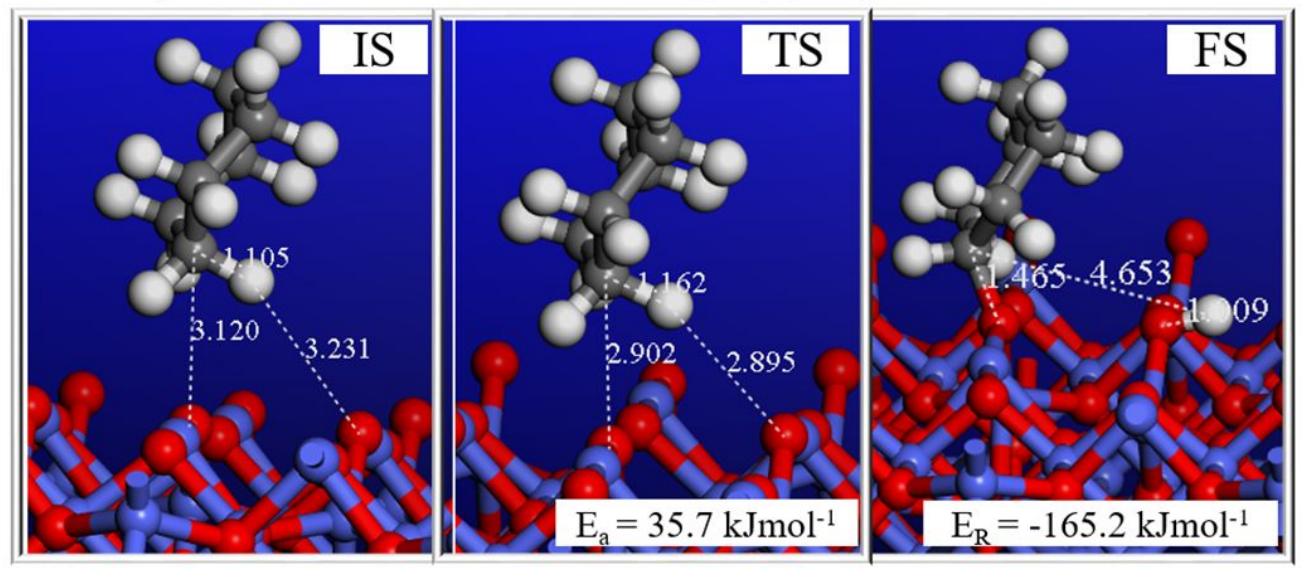

\section{Heterolytic mechanism on flat $\mathrm{Co}_{3} \mathrm{O}_{4}(110)$ surface}
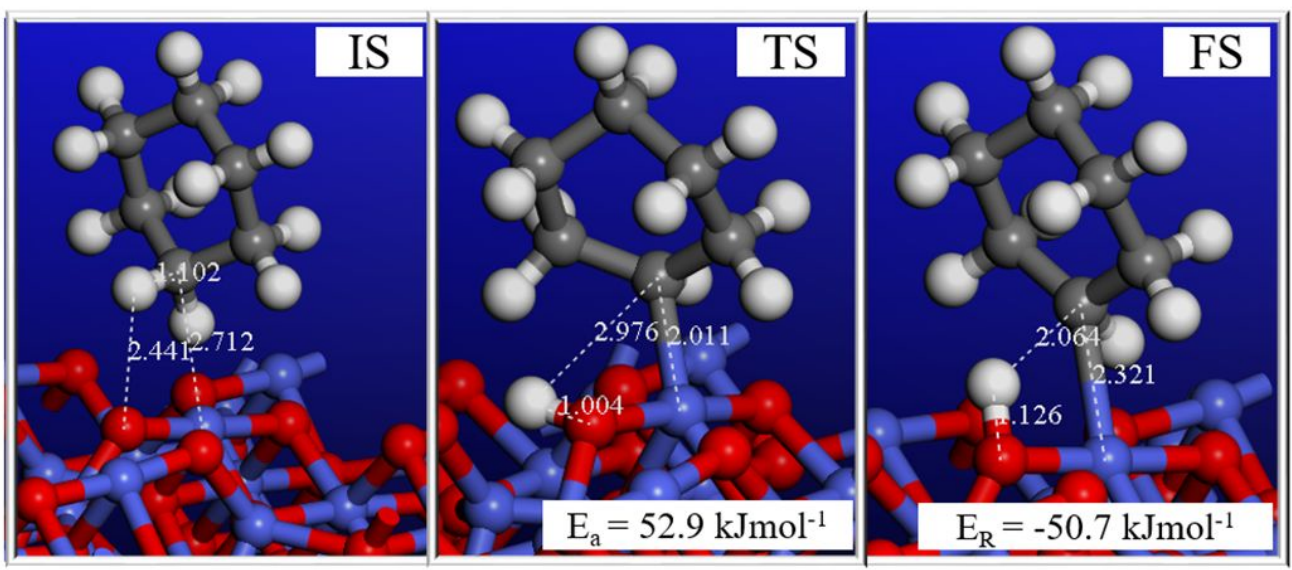

Figure S11. Activation barriers for the breakage of $\mathrm{Co}_{-}^{-}(\mathrm{CyOOH})$ and the formation of $\mathrm{CyCOOH}$ or $\mathrm{CyCO}$ radical through the hemolytic (above) and heterolytic (below) mechanisms. The bond lengths are shown in white texts $(\AA)$.

The activation of alkane $\mathrm{C}-\mathrm{H}$ bond over metal oxide surfaces has been divided into homolytic mechanism and heterotypic mechanism. ${ }^{\mathrm{S} 1-\mathrm{S} 4}$ The homolytic mechanism over $\mathrm{Co}_{3} \mathrm{O}_{4}(110)$ surface, involving cobalt-oxygen $\left(\mathrm{Co}^{*}-\mathrm{O}^{*}\right)$ site pairs, is proceeded via $\sigma$-bond metathesis, and the reaction path is: $\mathrm{C}_{6} \mathrm{H}_{12}+\mathrm{Co}^{*}-\mathrm{O}^{*} \rightarrow \mathrm{Co}^{*}-\mathrm{C}_{6} \mathrm{H}_{11}+\mathrm{O}^{*}-\mathrm{H}$. On the other hand, the reaction path of homolytic mechanism occurs with two surface oxygen atoms: $\mathrm{C}_{6} \mathrm{H}_{12}+2 \mathrm{O}^{*} \rightarrow \mathrm{O}^{*-} \mathrm{C}_{6} \mathrm{H}_{11}+$ $\mathrm{O}^{*}-\mathrm{H}$. The calculation results indicate that the homolytic mechanism $(35.7 \mathrm{~kJ} / \mathrm{mol})$ is more favorable than the heterolytic mechanism $(52.9 \mathrm{~kJ} / \mathrm{mol})$ on cyclohexane activation. 


\section{Co-O terminated surface}

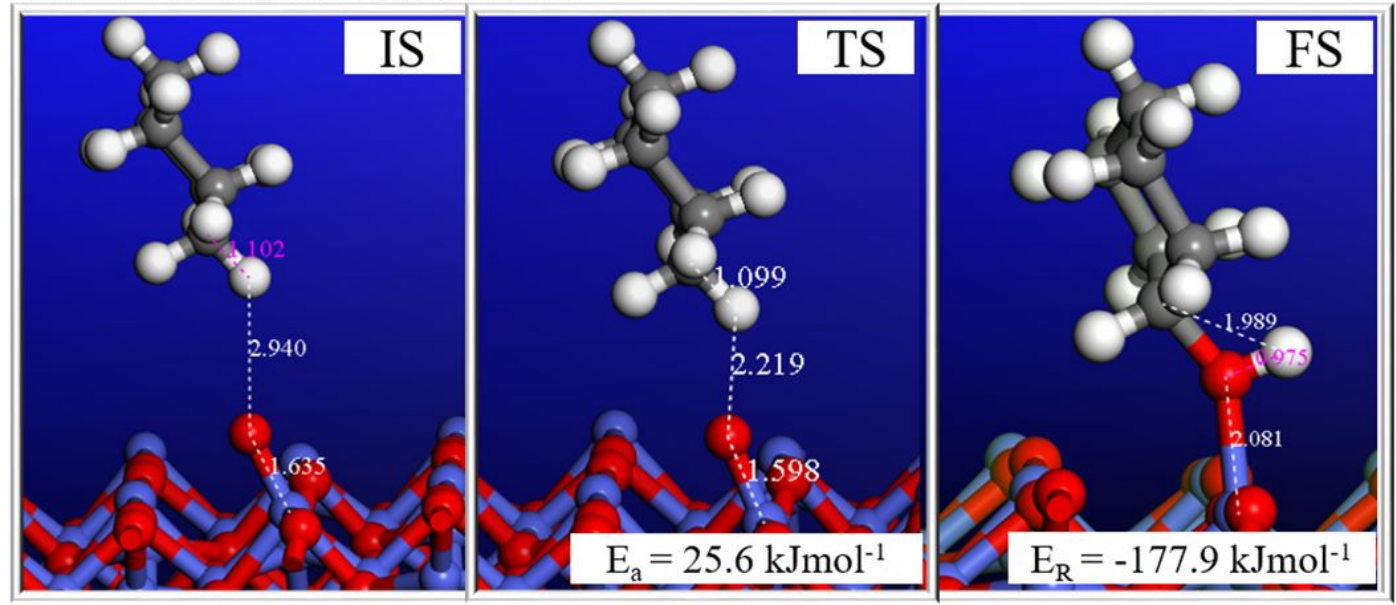

\section{$\mathrm{Co}(-\mathrm{O})_{2}$ terminated surface}

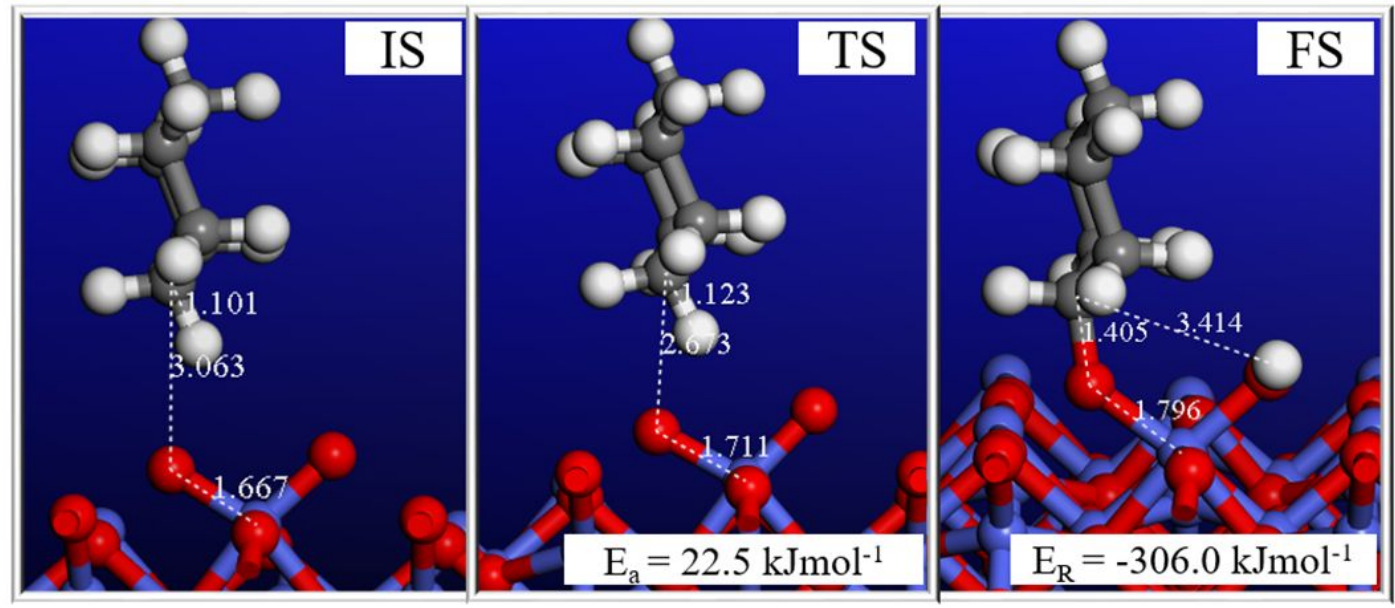

Figure S12. Activation barriers for the first C-H bond activation of cyclohexane over $\mathrm{Co}_{3} \mathrm{O}_{4}$ (111) surface terminated with one and two active oxygen atoms. The bond lengths are shown in white/purple texts $(\AA)$. 


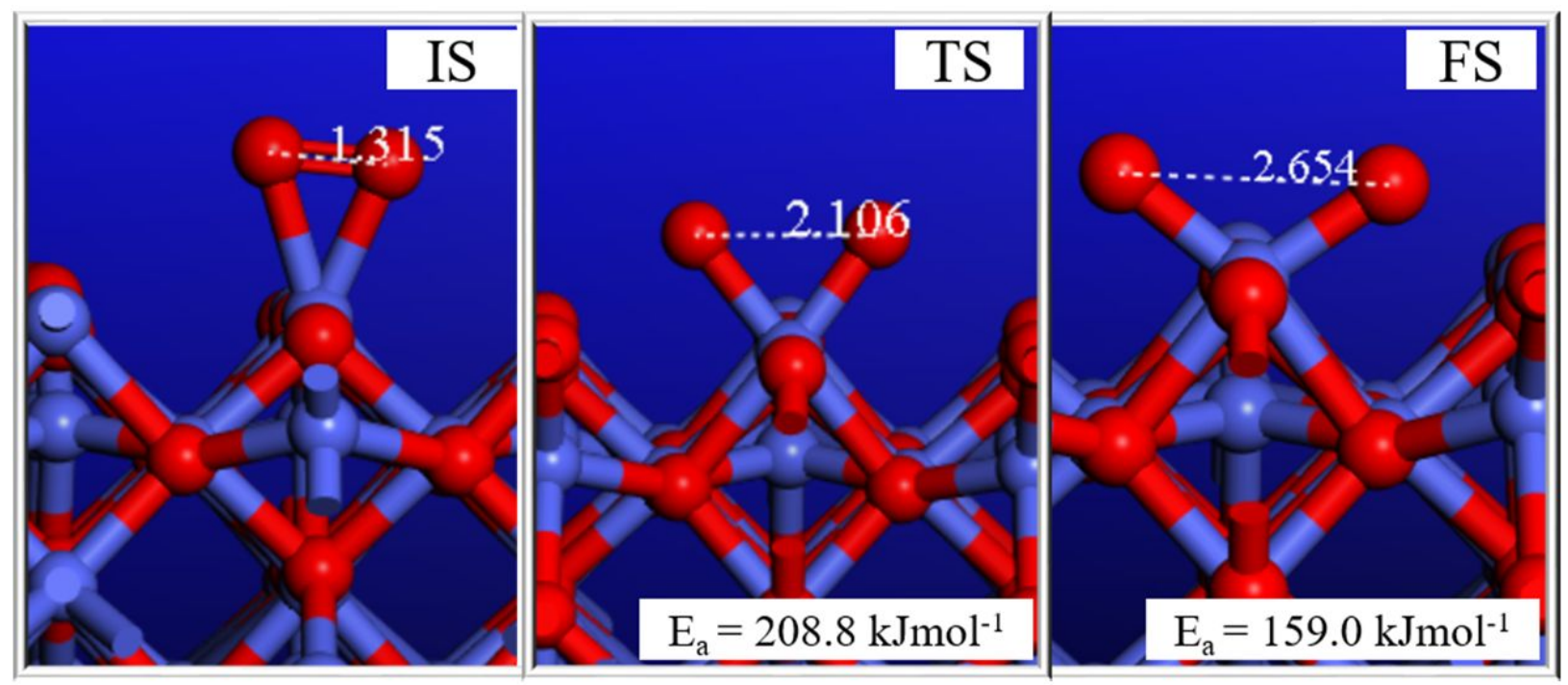

Figure S13. Activation barriers for the breakage of $\mathrm{O}_{2}$ molecule on $\mathrm{Co}_{3} \mathrm{O}_{4}(110)$ surface. The bond lengths are shown in white texts $(\AA)$. 
Table S1. $k$-Mesh and energy cutoff tests for the $\mathrm{Co}_{3} \mathrm{O}_{4}(110)$-B surface.

\begin{tabular}{cccc}
\hline & Parameter & $\begin{array}{c}\text { Structure energy per Co } \\
\text { atom }(\mathrm{eV} / \mathrm{Co} \text { atom })\end{array}$ & $\begin{array}{c}\text { Surface energy } \\
(\mathrm{eV} / \AA)\end{array}$ \\
\hline \multirow{4}{*}{-mesh } & $4 \times 3 \times 1$ & -14.17 & 0.110 \\
& $5 \times 3 \times 1$ & -14.17 & 0.110 \\
& $7 \times 4 \times 1$ & -14.16 & 0.111 \\
Energy & $450 \mathrm{eV}$ & -14.16 & 0.111 \\
cutoff & $500 \mathrm{eV}$ & -14.17 & 0.110 \\
& $550 \mathrm{eV}$ & -14.15 & 0.112 \\
\hline
\end{tabular}


Table S2. $k$-Mesh and energy cutoff tests for the $\mathrm{Co}_{3} \mathrm{O}_{4}(111)$ surface.

\begin{tabular}{cccc}
\hline & Parameter & $\begin{array}{c}\text { Structure energy per Co } \\
\text { atom }(\mathrm{eV} / \mathrm{Co} \text { atom })\end{array}$ & $\begin{array}{c}\text { Surface energy } \\
(\mathrm{eV} / \AA)\end{array}$ \\
\hline \multirow{4}{*}{$k$-mesh } & $2 \times 2 \times 1$ & -13.91 & 0.117 \\
& $4 \times 3 \times 1$ & -13.91 & 0.116 \\
& $5 \times 5 \times 1$ & -13.87 & 0.121 \\
Energy & $400 \mathrm{eV}$ & -13.89 & 0.119 \\
cutoff & $500 \mathrm{eV}$ & -13.91 & 0.116 \\
& $550 \mathrm{eV}$ & -13.86 & 0.122 \\
& & -13.94 & 0.113 \\
\hline
\end{tabular}


Table S3. Textural properties of as-prepared materials.

\begin{tabular}{cccc}
\hline Catalyst & $\mathrm{S}_{\mathrm{BET}}\left(\mathrm{m}^{2} \mathrm{~g}^{-1}\right)^{\mathrm{a}}$ & $\mathrm{S}_{\text {micro }}\left(\mathrm{m}^{2} \mathrm{~g}^{-1}\right)^{\mathrm{b}}$ & $\mathrm{S}_{\mathrm{ext}}\left(\mathrm{m}^{2} \mathrm{~g}^{-1}\right)^{\mathrm{c}}$ \\
\hline PMS-1a $(\mathrm{Si}: \mathrm{Co}=8: 1)$ & 409 & 9 & 400 \\
PMS-1 $(\mathrm{Si}: \mathrm{Co}=4: 1)$ & 391 & 8 & 383 \\
PMS-1b $(\mathrm{Si}: \mathrm{Co}=1: 1)$ & 321 & 6 & 315 \\
PMS-1c $(\mathrm{Si}: \mathrm{Co}=1: 4)$ & 45 & 9 & 35 \\
$250^{\circ} \mathrm{C} \mathrm{H} \mathrm{H}_{2}$ treated PMS-1 & 386 & 3 & 383 \\
$250^{\circ} \mathrm{C} \mathrm{H}_{2}$ treated PMS-1c & 134 & 44 & 90 \\
\hline
\end{tabular}

${ }^{a}$ Determined by multipoint BET method. ${ }^{b}$ Determined by $t$-method. ${ }^{c} t$-Plot external surface area. 
Table S4. Aerobic oxidation of cyclohexane over various catalysts. ${ }^{a}$

\begin{tabular}{ccccccc}
\hline Entry & Catalyst & $\begin{array}{c}\text { Co/cyclohexane } \\
(\mu \mathrm{mol} / \mathrm{mmol})\end{array}$ & $t(\mathrm{~h})$ & Conv. $(\%)^{\mathrm{b}}$ & Sel. $_{(\%)^{\mathrm{c}}}$ & $\mathrm{K} / \mathrm{A}$ \\
\hline 1 & $\mathrm{Co}_{3} \mathrm{O}_{4}$ & 1 & 3.0 & 1.8 & 98 & 1.4 \\
2 & $\mathrm{Co}_{3} \mathrm{O}_{4}$ & 4 & 3.0 & 3.1 & 98 & 1.6 \\
3 & $\mathrm{Co}_{3} \mathrm{O}_{4}$ & 8 & 3.0 & 6.9 & 96 & 4.5 \\
4 & $\mathrm{PMS}-1_{5}$ & 1 & 1.5 & 2.0 & 99 & 0.5 \\
6 & PMS-1 & 1 & 2.3 & 4.7 & 92 & 2.2 \\
7 & PMS-1 & 1 & 3.8 & 10.1 & 92 & 4.3 \\
8 & PMS-1 & 1 & 4.3 & 11.9 & 93 & 5.5 \\
9 & PMS-1 & 1 & 6.0 & 13.6 & 92 & 5.8 \\
\hline
\end{tabular}

aReaction conditions: $15 \mathrm{mmol}$ of cyclohexane, $5 \mathrm{~mL}$ of acetonitrile, $1.0 \mathrm{MPa}$ of oxygen, $120^{\circ} \mathrm{C}$. ${ }^{\mathrm{b}}$ Conversion $=\Sigma[$ product $] /[$ initial cyclohexane $] .{ }^{\mathrm{c} S e l e c t i v i t y}=\{[$ cyclohexanone $(\mathrm{K})]+$ [cyclohexanol (A)] $\} / \Sigma[$ product $]$.

With higher loading of $\mathrm{Co}_{3} \mathrm{O}_{4}$, which represents more active sites in the system, the conversion increased from 1.8 to $6.9 \%$. These results indicate that the accessibility and distribution of $\mathrm{CoO}_{\mathrm{x}}$ redox-active sites on the pore surfaces are particularly important for the catalytic reaction. 
Table S5. Aerobic oxidation of cyclohexane using various catalysts in the literature.

\begin{tabular}{|c|c|c|c|c|c|c|c|}
\hline Entry & Catalyst & $\mathrm{O}_{2}$ source & $T\left({ }^{\circ} \mathrm{C}\right)$ & $t(\mathrm{~h})$ & Conv. $(\%)$ & Sel. (\%) & Ref. \\
\hline 1 & $\mathrm{~g}-\mathrm{C}_{3} \mathrm{~N}_{4}$ & 10 bar $\mathrm{O}_{2}$ & 150 & 4.0 & 12 & - & S5 \\
\hline 2 & $\mathrm{Co}_{3} \mathrm{O}_{4}$ & $1.0 \mathrm{MPa} \mathrm{O}_{2}$ & 120 & 6.0 & 2.8 & 94.1 & S6 \\
\hline 3 & $\mathrm{NP} \mathrm{Co}{ }_{3} \mathrm{O}_{4}$ & $1.0 \mathrm{MPa} \mathrm{O}_{2}$ & 120 & 6.0 & 7.3 & 75.6 & S6 \\
\hline 4 & $\mathrm{Co} / \mathrm{Al}_{2} \mathrm{O}_{3}$ & $1.0 \mathrm{MPa} \mathrm{O}_{2}$ & 120 & 6.0 & 2.3 & 76.7 & S6 \\
\hline 5 & Co/ZSM-5 & $1.0 \mathrm{MPa} \mathrm{O}_{2}$ & 100 & 4.0 & 7.22 & 90.7 & S7 \\
\hline 6 & CoAlPO-5 & 1.0 MPa Air & 130 & 24.0 & 2.0 & 82.8 & S8 \\
\hline 7 & Fe-ZSM-5 & $1.0 \mathrm{MPa} \mathrm{O}_{2}$ & 100 & 4.0 & 6.47 & 79.6 & S7 \\
\hline 8 & FeAlPO-5 & 1.0 MPa Air & 130 & 24.0 & 6.6 & 51.7 & S8 \\
\hline 9 & MnAlPO-5 & 1.0 MPa Air & 130 & 24.0 & 6.2 & 61.3 & S8 \\
\hline 10 & Cu-SBA-15 & $1.0 \mathrm{MPa} \mathrm{O}_{2}$ & 120 & 5.0 & 9.5 & 81.2 & S9 \\
\hline 11 & $\mathrm{Au} / \mathrm{SBA}-15$ & $1.2-1.5 \mathrm{MPa}$ & 150 & 1.5 & 4.0 & 71 & S10 \\
\hline 12 & $\mathrm{Au}_{2} \mathrm{O}$ & 3 bar $\mathrm{O}_{2}$ & 140 & 17.0 & 1.9 & 69 & S11 \\
\hline 13 & $\mathrm{MgO}$ & 3 bar $\mathrm{O}_{2}$ & 140 & 17.0 & 1.0 & 98 & S11 \\
\hline 14 & $\mathrm{Ag}_{1} \mathrm{Pd}_{1} / \mathrm{MgO}$ & 3 bar $\mathrm{O}_{2}$ & 140 & 17.0 & 12 & 68 & S11 \\
\hline 15 & Ni-ZSM-5 & $1.0 \mathrm{MPa} \mathrm{O}_{2}$ & 100 & 4.0 & 2.53 & 70.0 & S7 \\
\hline 16 & Cr-ZSM-5 & $1.0 \mathrm{MPa} \mathrm{O}_{2}$ & 100 & 4.0 & 2.48 & 54.4 & S7 \\
\hline 17 & $\mathrm{Ti}_{60} \mathrm{Zr}_{10} \mathrm{Co}_{30}$ & $2.0 \mathrm{MPa} \mathrm{O}_{2}$ & 140 & 6.0 & 4.9 & 86.1 & S12 \\
\hline 18 & $\mathrm{Ce}_{0.5} \mathrm{Mn}_{0.5} \mathrm{O}_{\mathrm{x}}$ & 10 bar $\mathrm{O}_{2}$ & 120 & 4.0 & 10.5 & 84 & $\mathrm{~S} 13$ \\
\hline 19 & PMS-1 & $1.0 \mathrm{MPa} \mathrm{O} \mathrm{O}_{2}$ & 120 & 6.0 & 13.6 & 92 & $\begin{array}{l}\text { This } \\
\text { work }\end{array}$ \\
\hline
\end{tabular}




\section{References}

(S1) Tyo, E. C.; Yin, C.; Vece, M. D.; Qian, Q.; Kwon, G.; Lee, S.; Lee B.; Debartolo, J. E.; Seifert, S.; Winans, R. E.; Si, R.; Ricks, B.; Goergen, S.; Rutter, M.; Zugic, B.; Flytzani-Stephanopoulos, M.; Wang, Z. W.; Palmer, R. E.; Neurock, M.; Vajda, S. Oxidative Dehydrogenation of Cyclohexane on Cobalt Oxide $\left(\mathrm{Co}_{3} \mathrm{O}_{4}\right)$ Nanoparticles: The Effect of Particle Size on Activity and Selectivity. ACS Catal. 2012, 2 , 2409-2423.

(S2) Haber, J. Molecular Mechanism of Heterogeneous Oxidation — Organic and Solid State Chemists' Views. Stud. Surf. Sci. Catal. 2007, 110, 1-17.

(S3) Fu, G.; Xu, X.; Lu, X.; Wan, H. Mechanisms of Methane Activation and Transformation on Molybdenum Oxide Based Catalysts. J. Am. Chem. Soc. 2005, 127, 3989-3996.

(S4) Xu, X.; Faglioni, F.; Goddard, W. A. Methane Activation by Transition-Metal Oxides, $\mathrm{MO}_{\mathrm{x}}(\mathrm{M}=$ Cr, Mo, W; x =1, 2, 3). J. Phys. Chem. A 2002, 106, 7171-7176.

(S5) Li, X.-H.; Chen, J.-S.; Wang, X.; Sun, J.; Antonietti, M. Metal-Free Activation of Dioxygen by Graphene/g- $\mathrm{C}_{3} \mathrm{~N}_{4}$ Nanocomposites: Functional Dyads for Selective Oxidation of Saturated Hydrocarbons. J. Am. Chem. Soc. 2011, 133, 8074-8077.

(S6) Zhou, L.; Xu, J.; Miao, H.; Wang, F.; Li, X. Catalytic Oxidation of Cyclohexane to Cyclohexanol and Cyclohexanone over $\mathrm{Co}_{3} \mathrm{O}_{4}$ Nanocrystals with Molecular Oxygen. Appl. Catal. A 2005, 292, 223-228.

(S7) Yuan, H.-X.; Xia, Q.-H.; Zhan, H.-J.; Lu, X.-H. Su, K.-X. Catalytic Oxidation of Cyclohexane to Cyclohexanone and Cyclohexanol by Oxygen in a Solvent-Free System over Metal-Containing ZSM-5 Catalysts. Appl. Catal. A 2006, 304, 178-184.

(S8) Raja, R.; Sankar, G.; Thomas, J. M. Powerful Redox Molecular Sieve Catalysts for the Selective Oxidation of Cyclohexane in Air. J. Am. Chem. Soc. 1999, 121, 11926-11927. 
(S9) Gu, J.; Huang, Y.; Elangovan, S. P.; Li, Y.; Zhao, W.; Toshio, I.; Yamazaki, Y.; Shi, J. Highly Dispersed Copper Species within SBA-15 Introduced by the Hydrophobic Core of a Surfactant Micelle as a Carrier and Their Enhanced Catalytic Activity for Cyclohexane Oxidation. J. Phys. Chem. C 2011, 115, 21211-21217.

(S10) Hereijgers, B. P. C.; Weckhuysen, B. M. Aerobic Oxidation of Cyclohexane by Gold-Based Catalysts: New Mechanistic Insight by Thorough Product Analysis. J. Catal. 2010, 270, 16-25.

(S11) Liu, X.; Conte, M.; He, Q.; Knight, D. W.; Murphy, D. M.; Taylor, S. H.; Whiston, K.; Kiely, C. J.; Hutchings, G. J. Catalytic Partial Oxidation of Cyclohexane by Bimetallic Ag/Pd Nanoparticles on Magnesium Oxide. Chem. - A Eur. J. 2017, 23, 11834-11842.

(S12) Hao, J.; Wang, J.; Wang, Q.; Yu, Y.; Cai, S.; Zhao, F. Catalytic Oxidation of Cyclohexane over Ti-Zr-Co Catalysts. Appl. Catal. A 2009, 368, 29-34.

(S13) Zhang, P.; Lu, H.; Zhou, Y.; Zhang, L.; Wu, Z.; Yang, S.; Shi, H.; Zhu, Q.; Chen, Y.; Dai, S. Mesoporous $\mathrm{MnCeO}_{\mathrm{x}}$ Solid Solutions for Low Temperature and Selective Oxidation of Hydrocarbons. Nat. Commun. 2015, 6, 8446. 